\title{
Ligation-assisted endoscopic mucosal resection has high complete resection rate in rectal carcinoid tumor
}

Ming-Yao Su 1,2,3, $^{*}$ and Cheng-Tang Chiu 2,3,4 $^{2}$

\begin{abstract}
Aim: We aimed to compare the outcomes of different therapeutic modalities in rectal carcinoid tumors.

Method: We retrospectively collected 145 patients with rectal carcinoid tumors which were pathologically diagnosed from 2005/01/01 to 2016/12/31. We compared tumor size, complete resection rate and recurrent rate between different therapeutic modalities. Then, prospectively compared the treatment outcomes of 28 patients treated with ligation assisted endoscopic mucosal resection (LEMR) and 25 patients treated with endoscopic mucosal resection with cap (EMRC).

Result: The mean size of tumors was $6.5 \mathrm{~mm}(1-25 \mathrm{~mm})$, and the mean follow-up duration was 26 months (6-118 months). The therapeutic modalities included ligation-assisted endoscopic mucosal resection (LEMR) (25 tumors, 17\%), endoscopic mucosal resection (EMR) (31 tumors, 21\%), snare polypectomy (30 tumors, 21\%), biopsy forceps removal (46 tumors, 32\%) and surgical resection (13 tumors, 11\%), including 6 tumors treated with transanal endoscopic microsurgery (TEM) method. In view of pathologically complete resection rate, LEMR was highest (100\%), followed by surgical resection (85\%). However, EMR only had $42 \%$ pathologically complete resection rate. Besides, LEMR and surgical resection had no local recurrence and significantly higher clinically complete resection rate, compared to other treatments. For the further prospective study, complete resection was noted in 28 (100\%) patients in LEMR group and 13 (52\%) patients in EMRC group.
\end{abstract}

Conclusion: In the treatment of rectal carcinoid tumors, LEMR is safe and effective compared with traditional endoscopic treatments.

Keywords: Carcinoid, Neuroendocrine tumor, Endoscopic mucosal resection, Ligation

\section{Introduction}

Rectal carcinoid tumors are usually found incidentally during endoscopic examination. The typically endoscopic appearance of rectal carcinoid tumors is smooth, round, sessile elevations covered with normal-appearing or yellow-discolored mucosa [1]. The clinical characteristics

\footnotetext{
*Correspondence: doctorsmy54@gmail.com

${ }^{1}$ Department of Internal Medicine, New Taipei Municipal TuCheng Hospital, 6, Sec 2, Jincheng Rd, Tucheng Dist, New Taipei City, Taiwan, Republic of China

Full list of author information is available at the end of the article
}

of rectal carcinoid tumors include male predominance (1.6:1), small-sized tumors of $10 \mathrm{~mm}$ or less in detection (66.0\%), infrequent association with carcinoid syndrome $(0.7 \%)$, and relatively high 5 -year survival rate after removal (81.5\%) [2].

We can evaluate the malignant potential of rectal carcinoid tumors according to tumor size, endoscopic features, histological growth patterns, muscularis propria invasion, and lymphovascular invasion [1-3]. Among these parameters, primary tumor size is most simple and reliable predictor. Metastatic rate is less than 3\% 
in tumors $\leqq 10 \mathrm{~mm}$ in diameter and $5-15 \%$ in tumors between 11 and $20 \mathrm{~mm}$ in diameter. However, it is up to $80 \%$ in the tumor $>20 \mathrm{~mm}$ [4-6]. Previous studies suggested local resection for the carcinoid tumors less than $10 \mathrm{~mm}$ and confined to the submucosa because of low metastatic rate [7].

No matter endoscopic or surgical treatments, there were several methods for rectal carcinoid tumor resection. However, the therapeutic outcomes between different modalities are still unknown, and we aim to clarify the issue.

\section{Patients and methods}

\section{Endoscopic procedures}

\section{Endoscopic mucosal resection (EMR)}

After injecting saline solution beneath the tumor to lift it away from the muscularis propria, snare resection by serrated snare $30 \mathrm{~mm}$ in size with blended electrosurgical current was performed. For the retrospective analysis, the lesions were hot polypectomized or EMR, which were differentiated by submucosal fluid injection or no and used smooth snare or serrated snare. The EMR method had submucosal fluid injection combined with serrated snare.

\section{Endoscopic mucosal resection with cap (EMRC)}

Saline solution was injected into submucosal layer beneath the tumor to reduce the risks of perforation and resection margin involvement. The tumor was then aspirated into the cap, followed by snaring with the snare attached within the tip of cap. The snare resection was finally performed with blended electrosurgical current.

\section{Ligation-assisted endoscopic mucosal resection (LEMR)}

The procedure was carried out with a conventional single-channel endoscope (GIF-260, GIF-290, Olympus) with multi-band ligator device (Cook). It was then performed by following steps: (1) After identifying the lesion (Fig. 1A), we did submucosal saline solution injection beneath the tumor (Fig. 1B); (2) The tumor was aspirated into the ligation device, followed by deployment of the elastic band (Fig. 1C); (3) Snare resection with blended
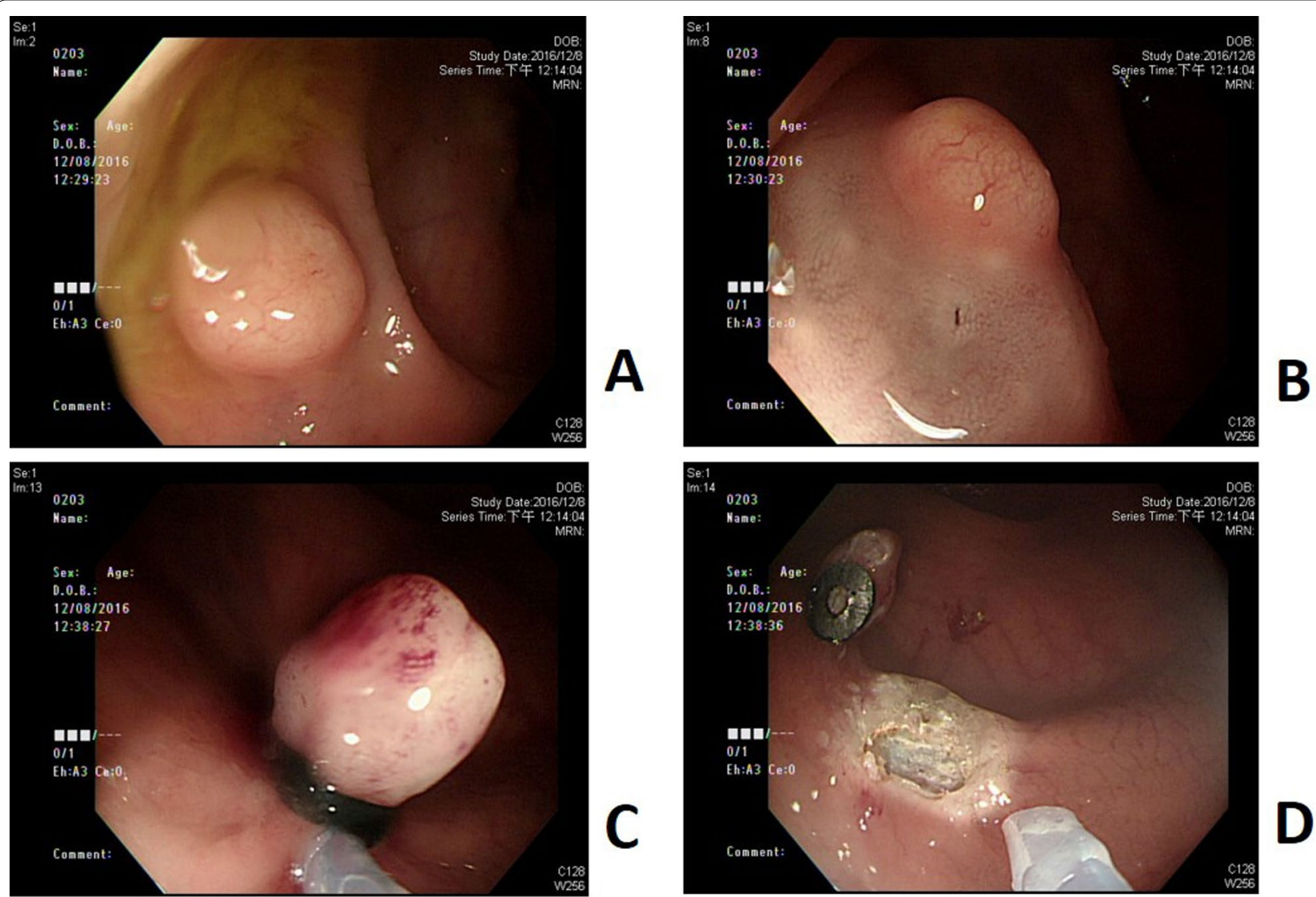

Fig. 1 The endoscopic procedure of LEMR. A Identify the lesion by endoscopy. B Submucosal saline solution was injected beneath the tumor to elevate it. C The lesion was then aspirated into the ligation device, followed by deployment of the elastic band. D Snare resection was performed below the band by using blended electrosurgical current 
electrosurgical current was performed below the ligation band (Fig. 1D).

\section{Histopathology evaluation}

After cutting the specimens into $2 \mathrm{~mm}$ slices, H\&E staining was performed to obverse the histopathology type, horizontal resected margin and vertical resected margin. The involvement of resection margin was decided according to the distance between tumor border and resection margin.

\section{Patients}

During 2005/01/01 to 2016/12/31, we retrospectively collected 145 rectal carcinoid tumor patients with pathological diagnosis in Chang Gung Memorial Hospital Linkou branch. All the patients had signed for the informed consent and this study was approved by the Chang Gung Medical Foundation Institutional Review Board number 201502259B0C501, and all methods were performed in accordance with the relevant guidelines and regulations. We exclude 31 patients who lost follow-up for the evaluation of recurrence. All enrolled patients had no clinical symptoms or signs of Carcinoid syndrome and tumors were found incidentally by colonoscopy check-up. The size of rectal carcinoid tumors and the methods of treatment were recorded. Therapeutic modalities were determined by the colonoscopists and proctologists during endoscopic examination. The primary outcomes included complete resection rate and local recurrent rate. Besides, the clinically complete resection rate (no recurrence found during follow-up) was also reviewed. All the polypectomy or EMR treatment were used hot coagulations, no cold snare used in this study. And all the procedures were performed by one experienced colonoscopist, who had performed more than 50,000 colonoscopies, 15,000 polypectomies and 2000 EMR procedures. Because the carcinoid tumors were subepithelial lesions, some of the resected margin of specimen were causative injury by coagulation and these cases were included with incomplete resection.

According to retrospective data, LEMR was $100 \%$ $(25 / 25)$ and ENR was $42 \%$ (13/31) complete resection rates, we conducted a prospective comparison of these two methods which need 25 patients each arm at least. We didn't consider of local recurrent rate for the primary end point. The patients were receiving EMR or LEMR therapy by randomized selection. There were 53 consecutive patients with rectal carcinoid tumors were randomized treated with LEMR (28 patients) and EMRC (25 patients) from $2017 / 1 / 1$ to $2019 / 6 / 30$. The complete resection rates were compared by this two groups of patients.
All data generated or analyzed during this study are included in this article. Please contacted Dr Ming-Yao Su if someone wants to request the data.

\section{Result}

The characteristics of these tumors from 145 patients are listed in Table 1. There were 57 males (39\%) and 88 females (61\%). The mean age at diagnosis was 49 years (range, 21-77 years). The mean size of tumor was $6.5 \mathrm{~mm}$ (range, 1-25 $\mathrm{mm}$ ) with average distance to anus about $6 \mathrm{~cm}$. Patients continued to undergo follow-up colonoscopy or sigmoidoscopy annually. The mean follow-up period was 26 months (range 6-118 months).

Thirty-one (21\%) tumors were treated with EMR, including 16 tumors were treated with EMR with cap (EMRC). Thirty (21\%) tumors were treated with snare polypectomy. Forty-six (32\%) tumors were treated with biopsy forceps removal. Thirteen (9\%) tumors were treated with surgical resection, including 6 tumors were treated with transanal endoscopic microsurgery (TEM) method. Twenty-five (17\%) tumors were treated with LEMR.

All the pathologic diagnoses were neuroendocrine tumor grade 1 with low mitotic rate $(<5$ in one high power view). No muscularis propria or lymphovascular invasion found in all complete resected specimens.

The treatment outcomes of rectal carcinoid tumors are listed in Table 2. The complete endoscopic resection rates were 100\% for LEMR, 97\% for EMR and 60\% for polypectomy. According to pathologic diagnosis, the LEMR group has the highest complete resection rate $(100 \%)$ based on pathologic evaluation, followed by surgical groups (85\%). The EMR group has about half complete resection rate $(42 \%)$ pathologically. While the polypectomy and biopsy groups had lowest complete resection rate based on pathologic evaluation (3 and $4 \%)$. The average resected margins for the complete resected tumors in each groups were $1.3 \mathrm{~mm}$ in LEMR group, $1.5 \mathrm{~mm}$ in surgical group, $0.4 \mathrm{~mm}$ in EMR group, $0.5 \mathrm{~mm}$ in polypectomy group and $0.1 \mathrm{~mm}$ in biopsy group, there significant difference between LEMR and

Table 1 The characteristics of patients and tumors

\begin{tabular}{llllll}
\hline & LEMR & EMR & Surgery & Polypectomy & Biopsy \\
\hline Patient number & 25 & 31 & 13 & 30 & 46 \\
Age & $51 \pm 16$ & $49 \pm 19$ & $43 \pm 21$ & $58 \pm 15$ & $44 \pm 21$ \\
Male (\%) & 40 & 39 & 46 & 37 & 39 \\
Tumor size (mm) & $6 \pm 3$ & $5 \pm 2$ & $7 \pm 5$ & $5 \pm 4$ & $6 \pm 4$ \\
BMl (mean) & 23.6 & 22.9 & 24.1 & 22.3 & 22.5 \\
Average distance & 6.7 & 6.3 & 6.5 & 5.8 & 6 \\
to anus (cm) & & & & & \\
\hline
\end{tabular}




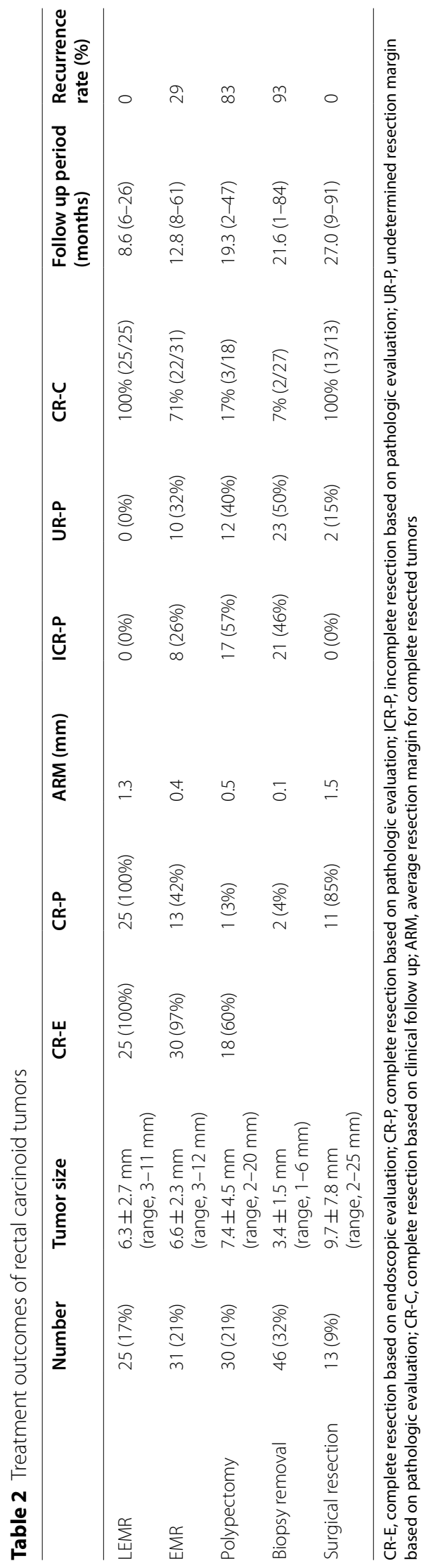


surgical group to other groups $(p<0.05)$. The 2 patients which had inadequate resection in surgical group were their lesions were too close to anus, so the surgeon consider to preserve the anal function so the resected margin were very close to tumor margin. Also, the clinically complete resection rate was high in the surgical resection and LEMR group (100\%). The local recurrent rate was higher in other groups (29\% in EMR group, $83 \%$ in polypectomy group and 93\% in biopsy group), as Table 2 . No major complications such as major bleeding or perforation occurred in current study. No metastatic lesion found during the period of follow- up. Except the surgical group, all patients received the endoscopic procedure at out-patient department.

We further compared the complete resection rate between LEMR and EMRC groups. 53 rectal carcinoid tumors were found incidentally by routine colonoscopy from $2017 / 1 / 1$ to $2019 / 6 / 30$ were randomized treated with LEMR 928 patients) and EMRC 925 patients) prospectively. No differences noted for the patients' characteristics included sex, age and tumor size for this two groups of patients (Table 3). However, the complete resection rate was $100 \%$ in LEMR group and $52 \%$ in EMRC group $(p<0.005)$.

\section{Discussion}

Several features have been reported as possible predictors for outcomes in patient with rectal carcinoid tumors. The features associated with a poor prognosis include large size, deep invasion, lymphovascular invasion, and elevated mitotic rate $[8,9]$. However, the size of the primary tumor was a simple and reliable factor for predicting the risk of metastasis. Therefore, rectal carcinoid tumors are considered as a good candidate for local excision, including endoscopic or transanal resection. When the tumor was less than $10 \mathrm{~mm}$ in diameter, without atypical features and confined to the submucosal layer, the possibility of lymphovascular invasion or distant metastasis was very rare [10-12]. Various methods

Table 3 The characteristics of patients treated with LEMR and EMR

\begin{tabular}{llll}
\hline & LEMR & EMR & $\boldsymbol{p}$ value \\
\hline Patient number & 28 & 25 & NS \\
Age & $48 \pm 12$ & $50 \pm 14$ & NS \\
Male (\%) & 50 & 52 & NS \\
Tumor size (mm) & $6 \pm 4$ & $5 \pm 3$ & NS \\
BMl (mean) & 24.2 & 23.9 & NS \\
Average distance to anus (cm) & 6.2 & 6.4 & NS \\
Complete resection rate (\%) & 100 & 52 & $p<0.005$ \\
\hline
\end{tabular}

of endoscopic resection for rectal carcinoid tumors have been developed. Endoscopic mucosal resection with or without cap is considered as an effective method for complete resection.

During conventional endoscopic mucosal resection (EMR), the lesions were elevated by injecting saline (or other solute) into the underlying submucosal layer and then snared and resected using a blend current [13]. Due to the submucosal nature of rectal carcinoid tumors and sharpness of snare, conventional EMR is more likely to associated with incomplete resection margins $[14,15]$. Another alternative to conventional EMR involves suctioning the area raised by solute injection into a transparent cap (EMR-C) and either cleaving the lesion directly or banding it, with subsequent snare resection and retrieval. Some pilot studies suggest that these methods may be more effective [16-25]. However, most of these studies only enrolled limited number of cases. In our series, the complete histopathologically resection rate by EMR or EMRC method is only $42 \%$. The reason may be related to the characteristic of snare, which is very thin and sharp, and would cut through the tumor and resulted in incomplete resection. The recurrence lesions were found as soon as one month after local management, so, we suggest $\mathrm{f} / \mathrm{u}$ colonoscopy at least one month later after incomplete resection procedures.

During ligation-assisted endoscopic mucosal resection (LEMR), a rubber band was used to ligate the tumor before the procedure. Because the rubber band can follow the curve of tumor, it would be tight below the lesion strongly and facilitate further en-bloc snaring and resection. In our study, by this LEMR method, all lesions can be resected completely by histopathology evaluation with adequate resected margin.

Complete resection of carcinoid tumors of the rectum remains be difficult by conventional polypectomy or biopsy method. In our study, the rate of complete resection was only $3-4 \%$, which is inferior to the results obtained by EMR, EMRC or LEMR. According to our current study, we suggest that biopsy removal or polypectomy are not adequate treatment method for rectal submucosal lesion. And the lesion should be resected by other advanced procedures, such as LEMR or surgery, to achieve high complete resection rate. The 2 patients which had inadequate resection in surgical group were their lesions were too close to anus, so the surgeon consider to preserve the anal function so the resected margin were very close to tumor margin, so for the lesions close to anus may choose LEMR for the treatment option.

Although the complete resection rate was not high for EMR or EMRC, the local recurrence was not high in our study. Possible reasons for this observation include: (1) electrocoagulation may have caused necrosis of 
the peripheral margins of the resected specimens; (2) the behavior of these carcinoid tumors was indolent; and (3) the follow-up period was too short for tumor recurrences.

Further prospectively analysis of complete resection rates compared with LEMR and EMRC groups, which showed that significant difference between this two groups (100\% in LEMR and 52\% in EMRC group, $p<0.005)$. Similar result was shown by our previous retrospective analysis.

For some lesions more than $2 \mathrm{~cm}$ in size, LEMR may be not suitable for the limitation of cap's size, while endoscopic mucosal dissection (ESD) may be an alternative treatment, but ESD need more learning curve and we will start another study for the treatment outcomes of ESD for rectal carcinoid tumors.

In the past, all recurrent cases were refer to surgery for further management; however, after the retrospective analysis data noted, we treated the later cases by LEMR with good results (100\% complete resection rate).

In conclusion, for rectal carcinoid tumors, LEMR had highest complete resection rate with adequate resected margin and no local recurrence during follow-up even compared with EMR. Although surgery also had high complete resection rate and no local recurrence found, it takes the cost of admission and the risk of anesthesia. LEMR is a safe and effective modality for treating rectal carcinoid tumors.

\section{Acknowledgements \\ This paper had partly presented at DDW 2016, and published at GIE 83(5) suppl AB22-9, 2016 [26].}

\section{Authors' contributions}

Dr M-YS: Substantial contributions to the conception or design of the work; analysis, and interpretation of data of data for the work; Drafting the article or revising it critically for important intellectual content; Final approval of the version to be published. Agreement to be accountable for all aspects of the work. Dr C-TC: Substantial contributions to the interpretation of data of data for the work; Revising it critically for important intellectual content; Final approval of the version to be published. Agreement to be accountable for all aspects of the work. Both authors read and approved the final manuscript.

\section{Funding}

This study has no funding support.

\section{Availability of data and materials}

Please contacted Dr Ming-Yao Su if someone wants to request the data.

\section{Declarations}

\section{Ethics approval and consent to participate}

All the patients had signed for the informed consent and this study was approved by the Chang Gung Medical Foundation Institutional Review Board number 201502259B0C501, and all methods were performed in accordance with the relevant guidelines and regulations.

\section{Consent for publication}

The authors agree for the consent for publication.

\section{Competing interests}

The authors has no conflict of interest.

\section{Author details}

${ }^{1}$ Department of Internal Medicine, New Taipei Municipal TuCheng Hospital, 6, Sec 2, Jincheng Rd, Tucheng Dist, New Taipei City, Taiwan, Republic of China. ${ }^{2}$ Department of Gastroenterology and Hepatology, Chang Gung Memorial Hospital Linkou Branch, Taoyuan, Taiwan. ${ }^{3}$ Chang Gung University College of Medicine, Taoyuan, Taiwan. ${ }^{4}$ Taiwan Association for the Study of Small Intestinal Diseases, Taoyuan, Taiwan.

Received: 2 August 2021 Accepted: 6 December 2021

Published online: 13 December 2021

\section{References}

1. Shim K-N, Yang S-K, Myung S-J. Atypical endoscopic features of rectal carcinoids. Endoscopy. 2004;36:313-6.

2. Soga J. Carcinoids of the rectum: an evaluation of 1271 reported cases. Surg Today. 1997;27:112-9.

3. Burke M, Shepard N, Mann CV. Carcinoid tumor of the rectum and anus Br J Surg. 1987;74:358-61.

4. Fitzgerald SD, Meagher AP, Moniz-Pereira P. Carcinoid tumor of the rectum: DNA ploidy is not a prognostic factor. Dis Colon Rectum. 1996;39:643-8.

5. Mani S, Modlin IM, Ballantyne G. Carcinoids of the rectum. J Am Coll Surg. 1994;179:231-48

6. Naunheim KS, Zeitels J, Kaplan EL. Rectal carcinoids: treatment and prognosis. Surgery. 1983;94:670-6.

7. Fahy BN, Tang LH, Klimstra D. Carcinoid of the Rectum Risk Stratification (CaRRS): a strategy for preoperative outcome assessment. Ann Surg Oncol. 2007;14:396-404.

8. Mashimo Y, Matsuda T, Uraoka T. Endoscopic submucosal resection with a ligation device is an effective and safe treatment for carcinoid tumors in the lower rectum. J Gastroenterol Hepatol. 2008;23:218-21.

9. lishi $\mathrm{H}$, Tatsuta M, Yano H. More effective endoscopic resection with a two-channel colonoscope for carcinoid tumors of the rectum. Dis Colon Rectum. 1996:39:1438-9.

10. Kobayashi K, Katsumata T, Yoshizawa S. Indications of endoscopic polypectomy for rectal carcinoid tumors and clinical usefulness of endoscopic ultrasonography. Dis Colon Rectum. 2005;48:285-91.

11. Higaki S, Nishiaki M, Mitani N. Effectiveness of local endoscopic resection for rectal carcinoid tumors. Endoscopy. 1997;29:171-5.

12. Shirouzu K, Isomoto H, Kakegawa T. Treatment of rectal carcinoids. Am J Surg. 1990;160:262-5.

13. Matsui K, Iwase T, Kitagawa M. Small polypoid-appearing carcinoid tumors of the rectum: clinicopathologic study of 16 cases and effectiveness of endoscopic treatment. Am J Gastroenterol. 1993;88:1949-53.

14. Charles B, Irina J, Edvardas K. Band-snare resection of small rectal carcinoid tumors. Gastrointestinal Endosc. 1999:50:582-5.

15. Nagai T, Torishima H, Nakashima H. Saline-assisted endoscopic resection of rectal carcinoids: cap aspiration method versus simple snare resection. Endoscopy. 2004;36:202-5.

16. Ono A, Fujii T, Saito Y. Endoscopic submucosal resection of rectal carcinoid tumors with a ligation device. Gastrointestinal Endosc. 2003; 57:585-7

17. Hurlstone DP, Cross SS, Sanders DS. 20-MHz high-frequency endoscopic ultrasound-assisted endoscopic mucosal re- section for the colorectal submucosal lesions: a prospective analysis. J Clin Gastroenterol. 2005;39:555-6.

18. Waxman I, Saitoh Y, Raju GS. High-frequency probe EUS- associated endoscopic mucosal resection: a therapeutic strategy for submucosal tumors of the Gl tract. Gastrointest Endosc. 2002;55:44-9.

19. Memon MA, Nelson H. Gastrointestinal carcinoid tumors: current management strategies. Dis Colon Rectum. 1997;40:1101-8.

20. Stinner B, Kisker O, Zielke A, Rothmund M. Surgical management for carcinoid tumors of small bowel, appendix, colon, and rectum. World J Surg. 1996;20:183-8.

21. Shields CJ, Winter DC. Carcinoid tumors of the rectum: a multi-institutional international collaboration. Ann Surg. 2010;252:750-5. 
22. Godwin JD. Carcinoid tumors: an analysis of 2837 cases. Cancer. 1975;36:560-9.

23. Quan SHQ, Bader G, Berg JW. Carcinoid tumors of the rectum. Dis Colon Rectum. 1964;7:197-206.

24. Caldarola VT, Jackman RJ, Moertel CG. Carcinoid tumors of the rectum. Am J Surg. 1964;107:844-9.

25. Ramage JK, Goretzki PE, Manfredi R. Consensus quidelines for the management of patients with digestive neuroendocrine tumors: welldifferentiated colon and rectum tumor/ carcinoma. Neuroendocrinology. 2008:87:31-9.

26. SU MY, Chiu CT. Treatment outcomes of rectal carcinoid tumors. GIE. 2016;83(5 suppl):AB228-9.

\section{Publisher's Note}

Springer Nature remains neutral with regard to jurisdictional claims in published maps and institutional affiliations.

- fast, convenient online submission

- thorough peer review by experienced researchers in your field

- rapid publication on acceptance

- support for research data, including large and complex data types

- gold Open Access which fosters wider collaboration and increased citations

- maximum visibility for your research: over 100M website views per year

At BMC, research is always in progress.

Learn more biomedcentral.com/submissions 